\title{
Living wills: would sick people change their minds?
}

\author{
Janette M. Potter, David Stewart and George Duncan ${ }^{1}$ \\ Victoria Geriatric Unit, Victoria Infirmary NHS Trust, Mansionhouse Road, Glasgow and ${ }^{1}$ Biggart \\ Hospital, Prestwick, Ayrshire, Scotland
}

\begin{abstract}
Summary: Patients admitted acutely to a geriatric medical unit were interviewed on admission about their opinions on cardiopulmonary resuscitation (CPR). They underwent a general examination and their mental health was documented by completing the geriatric depression scale. Those who did not wish CPR in the event of a cardiac arrest were questioned again on recovery.

Of 216 patients admitted, only three objected to answering the questions and after the other exclusion criteria were applied, 100 patients were included in the study. A total of $92 \%$ of patients wished CPR in the event of a cardiac arrest. The $8 \%$ who did not wish CPR contained more people scoring high on the geriatric depression scale. After recovery, three of that eight had changed their minds and wished CPR if required.

Patients who are acutely unwell may make decisions that are influenced by their condition at this point in time and it is important to recognize that these decisions may not be maintained. In this study, consultant geriatricians did not reflect their patients' desires in making decisions about who should receive CPR if required.
\end{abstract}

\section{Introduction}

Studies show that advanced age does not preclude a successful outcome from cadiopulmonary resuscitation (CPR). ${ }^{\prime}$ Directives from the Department of Health and the British Geriatric Society encourage doctors to document whether patients are appropriate for CPR, taking into account relatives' views where relevant. This is often difficult in practice and is further complicated by the fact that the patient may be unable to express their views when decisions have to be made. We know, however, that patients' wishes in this regard are not always reflected in the views of their close relatives. ${ }^{2}$ Living wills and advance directives have been considered, in principle, to be useful by physicians, patients and relatives. ${ }^{3}$ Despite this apparent enthusiasm they are rarely used in the USA or in the UK. It is estimated that their use is only $27 \%$ at the highest ${ }^{4}$ (and Dr J.M.A. Burns, unpublished work). Elderly patients' views on CPR have been considered when the patients had recovered from their illness and were about to be discharged home, and in patients who are well and living in the community. ${ }^{5,6}$ This, however, is of little practical use. The views of patients while still unwell have not been reported and are of much more relevance to clinical practice.

Correspondence: J.M. Potter, M.B., Ch.B., M.R.C.P. (U.K.).

Accepted: 9 June 1994 been reported and are of much more relevance to clinical practice.

Living wills are usually made when death or serious illness is an abstract concept, and it is possible that, when it becomes a more concrete threat, a patient's views may change. ${ }^{3} A$ living will can be altered at any time during illness, so long as the patient remains physically and mentally competent to do so. However, there has been little research into the effect of illness on a patient's feelings about ongoing care. Thus should a patient with a living will suddenly become unwell, the freedom for him or her to change his or her mind about the management of their illness, and ultimately whether or not he ought to be resuscitated, may be removed.

Given the complexity of interacting factors in the acute situation, which might influence decisions on the appropriateness of a patient for CPR, we feel it would be important to know the patient's own opinion at that time. It is possible that a patient's opinion during illness might be influenced by the effect of his physical condition on his psychological well being, and therefore we have attempted to take this into consideration.

Thus we have attempted to define whether patients admitted acutely to a geriatric medical unit, wish resuscitation if required, and whether their views are influenced by their physical and psychological well-being. 


\section{Patients and methods}

Patients over 65 years old with a variety of medical illnesses, admitted directly from home as emergencies, were assessed on the morning after hospital admission. Cognitive function was assessed by the Abbreviated Mental Test (AMT) and consent was sought. Depressive symptoms were documented by using the Geriatric Depression Scale (GDS), and a patient was considered to show evidence of depressive symptoms if he or she had a score of $>11$ out of $30 .^{7}$ Moribund patients and those with an AMT $<7$ out of 10 were excluded. Patients were then asked a series of questions by a doctor (see Table I). All patients were given a standard layman's explanation of CPR. The likely success of CPR in individual cases was not discussed. The consultant responsible for that patient's care was then asked whether they considered CPR to be appropriate without knowing the patient's views. The concurrence of views between patients and consultants was compared using McNemar's test, and the number of patients scoring $>11 / 30$ for GDS in those wishing CPR or no CPR were compared by chi-squared analysis. Patients answering no to question 5 were requestioned just prior to discharge.

\section{Results}

A total of 100 patients were included. Their mean age was 81 years (range 63-93). Of 216 admissions, 116 were excluded, only three objected to answering the questions, 93 were cognitively impaired, eight had communication difficulties and 12 were moribund. By the nature of the study and the exclusion criteria, all the participants were medically unwell. Twenty-two patients had evidence of depression.

A total of 92 patients wanted CPR if required. The eight patients who did not were requestioned after recovery and three of them then wanted CPR. Of the eight patients who did not want CPR, five $(63 \%)$ scored $>11$ on the GDS compared with 17 of the $92(18 \%)$ patients who wanted CPR $(P=0.02)$.

\section{Table 1 Patients questionnaire}

1. Do you think every elderly patient who has a cardiac arrest should have CPR?

2. Do you think no elderly patients should have CPR?

3. Would you set an age limit for CPR? If so what?

4. Would you set a limit based on pre-existing disability? If yes, for physical impairment and/or mental disability.

5. Would you wish CPR if you had a cardiac arrest?
A total of 89 patients felt that all elderly patients should be resuscitated, if necessary; $17 \%$ of these patients thought that there should be an age limit for CPR. The numbers opting for each age limit were as follows: $6>100$ years, $6>90$ years, $3>80$ years, and $2>70$ years.

A total of 38 patients thought that there should be a limit based on quality of life. Three for physical disability and 13 for mental disability, 22 for either. This group included 10 patients who also thought that there should be an upper age limit.

Consultants thought $80 \%$ of the patients were appropriate for CPR. Of the 20 patients not considered suitable for CPR, 18 had requested it if required. Those patients not wishing CPR would have been resuscitated in six out of eight cases, confirming significant discordance $(P<0.05)$.

\section{Discussion}

The majority of unwell elderly patients admitted acutely to hospital were happy to discuss their views on CPR, with only three patients of a total 216 abstaining. This is in keeping with previous studies of elderly outpatients and in-patients who are not acutely unwell. ${ }^{1.5}$ It is interesting to note that acute illness does not alter this. The great majority of elderly patients who were not cognitively impaired wished to have resuscitation if necessary. This result is in contrast to previous studies considering elderly people's views while they are well. ${ }^{5.6}$ The reason for this is unclear, but it may be related to the fact that their circumstances were no longer hypothetical. Those few patients who did not wish to be resuscitated were more likely to be depressed and it is concerning to note that some of them changed their views during hospitalization. This finding casts doubt on the belief that a decision a patient may make while they are either well or at a particular stage in their illness is necessarily representative of their decision later in their illness.

Fried et $a .^{3}$ looked retrospectively at decisions made by community-dwelling unwell elderly patients during what turned out to be their final illness. Their findings supported our concern that advance directives and living wills inhibit the ongoing liaison between doctor and patient, which allows the patient to make decisions based on the actual circumstances they find themselves in and how they feel at that time. We did not attempt to discuss the prognosis of or likelihood of success of CPR in individual patients, although Murphy et $a l^{7}$ have recently shown that elderly people are able to consider these factors in the process of making decisions about CPR.

Since it is recognized that neither relatives' nor consultants' views necessarily reflect patient's 
desires, ${ }^{2,8}$ and we have confirmed discordance between the views of consultants and patients, we conclude that elderly patients ought to be given the opportunity to express their opinions on CPR as a

\section{References}

1. Gulati, R.S., Bhan, G.L. \& Horan, M.A. Cardiopulmonary resuscitation of old people. Lancet 1983, ii: 267-269.

2. Seckler, A., Mejer, D., Mulvihill, M. \& Cammer Paris, B. Substituted judgement: how accurate are proxy predictions? Ann Intern Med 1991, 115: 92-98.

3. Fried, T.R. \& Gillick, M.R. Medical decision making in the last six months of life: choices about limitation of care. JAGS 1994, 42: 302-307.

4. Emanuel, L. The health care directive: learning how to draft advance care documents. JAGS 1991, 39: 1221-1228.

5. Wagner, A. Cardiopulmonary resuscitation in the aged-a prospective survey. $N$ Engl J Med 1984, 310: 1129-1130. matter of routine. Such decisions ought to be subject to regular review and the fact that few $\underset{\complement}{\mathbb{\Phi}}$ patients actually compose a living will may not be $c$. inappropriate.

6. Gunasekara, N.P.R., Tiller, D.J., Clements, L.T. \& Bhat- $\varrho$ tacharya, B.K. Elderly patients' views on CPR. Age Ageing 1986, 15: 358-364.

7. Murphy, D.J., Burrows, D., Santilli, S., Kemp, A.W., Tenner, $\overrightarrow{0}$ S., Kreling, B. \& Teno, J. The influence of the probability of survival on patients preferences regarding CPR. $N$ Engl J Med $\vec{\omega}$ 1994, 330: 545-549.

8. Liddle, J., Gilleard, C. \& Neil, A. Elderly patients' and their relatives' views on CPR. Lancet 1993, 342: 1055. 